\title{
Structure of hadron resonance with nearby CDD zero
}

\section{Yuki Kamiya*}

Yukawa Institute for Theoretical Physics, Kyoto University, Kyoto 606-8502, Japan

E-mail: vuki.kamiyadyukawa.kyoto-u.ac.ip

\section{Tetsuo Hyodo}

Yukawa Institute for Theoretical Physics, Kyoto University, Kyoto 606-8502, Japan

E-mail: hyododyukawa.kyoto-u.ac.ip

We discuss the method to investigate the origin of hadron resonances from the analytic structure of the scattering amplitude. Recently, there have been discussions on the relation between the hadron structure and the position of the CDD (Castillejo-Dalitz-Dyson) zero, which is defined as the zero of the amplitude. To study the relation between the position of the CDD zero and the internal structure of the hadrons, we consider the zero coupling limit (ZCL) of the coupled channel scattering amplitude, where the couplings among different channels are turned off. With the behavior of the pole in the ZCL, we reveal the origin of the eigenstate. By studying the behavior of the eigenstate poles and the CDD zeros in the ZCL, it is shown that the origin of the pole accompanied by a nearby CDD zero is the dynamics of the hidden channel. As an application, we show that the High-mass (Low-mass) pole of the $\Lambda(1405)$ baryon has the dynamical origin of the $\bar{K} N(\pi \Sigma)$ channel.

XVII International Conference on Hadron Spectroscopy and Structure - Hadron2017

25-29 September, 2017

University of Salamanca, Salamanca, Spain

\footnotetext{
*Speaker.
} 


\section{Introduction}

The emergence of the many candidates of the exotic hadrons in the recent experiment [W] requires the method to clarify the internal structure of hadrons with experimental analyses. With the experimental analyses, we can obtain the scattering amplitude on the real energy region, which can be analytically continued to the complex energy axis [ [ [] . In the scattering amplitude, the eigenstate is expressed as the pole. Thus by deriving the relation between the structure of the amplitude around the pole energy and the internal structure of the eigenstate, we can discuss the structure of hadron resonances directly from the experimental data.

On the other hand, the importance of the zero of the scattering amplitude, which is called the

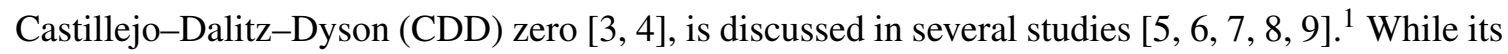
importance is recognized, the direct relation between the structure of the state and the CDD zero

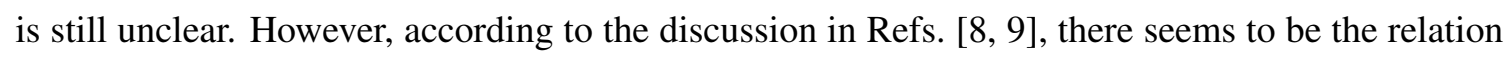
between the position of the CDD zero and the internal structure of the hadron resonances.

In this paper, we discuss the relation between the structure of the hadron resonances and positions of the poles and CDD zeros. First, we show that the origin of the eigenstate is specified with the behavior of the coupled channel scattering amplitude in the zero coupling limit (ZCL), where the inter-channel coupling is switched off. Next, with the consideration on the topological nature of the scattering amplitude, we show the relation between the origin of the hadron resonances and the position of the CDD zero. Finally, we show the application to the $\Lambda(1405)$ baryon. All the contents in this paper is based on Ref. [ए]].

\section{Zero coupling limit and origin of eigenstate}

In this section, we show how to extract the information of the internal structure of hadron resonances from the coupled-channel scattering amplitude in a given partial wave. To this end, we focus on the positions of the pole $E_{\text {pole }}$ and CDD zero $E_{\mathrm{CDD}}$ of the diagonal component of the scattering amplitude $\mathscr{F}_{i i}(E)$. We notice that the eigenstate pole emerges at the same energy $E_{\text {pole }}$ in all the components of the coupled channels. On the other hand, the existence and the position of the CDD zero, which is defined as the zero of the amplitude $\mathscr{F}_{i i}\left(E_{\mathrm{CDD}}\right)=0$, is channel dependent.

Taking the zero coupling limit (ZCL), where the coupling between different channels are switched off [ㄴ], [2, [3]], we can specify the origin of the eigenstate as follows. Decreasing the coupling, the eigenstate pole moves in the complex energy plane. In the exact ZCL, the pole remains only in the one of the components, which is considered as the origin of the eigenstate. If the pole remains in the amplitude of the focused channel in the ZCL, the dynamics of the focused channel is the origin of the eigenstate. In contrast, if the pole decouples from the amplitude, the dynamics of the focused channels is not the origin. In the following, we show that the position of the CDD zero is related to the origin of the eigenstate.

To derive the relation between the origin of the eigenstate and the position of the eigenstate, let us consider a single-channel scattering problem coupled to a bare state, utilizing the nonrelativistic

\footnotetext{
${ }^{1}$ The CDD zero is often called as "CDD pole" because it is the pole of the inverse amplitude. In this paper, to avoid the confusion with the eigenstate pole, we call it CDD zero.
} 
effective field theory introduced in Ref. [Q] :

$$
\begin{gathered}
H=\int d^{3} \boldsymbol{x}\left[\frac{1}{2 M} \nabla \psi^{\dagger} \cdot \nabla \psi+\frac{1}{2 m} \nabla \phi^{\dagger} \cdot \nabla \phi+\frac{1}{2 M_{0}} \nabla B_{0}^{\dagger} \cdot \nabla B_{0}+\omega_{0} B_{0}^{\dagger} B_{0}\right. \\
\left.+g_{0}\left(B_{0}^{\dagger} \psi \phi+\phi^{\dagger} \psi^{\dagger} B_{0}\right)+v_{0} \psi^{\dagger} \phi^{\dagger} \phi \psi\right],
\end{gathered}
$$

with $\hbar=1$. By solving the Lippmann-Schwinger equation, we obtain the exact on shell $T$-matrix:

$$
t(E)=\frac{v_{0}\left(E-\omega_{0}\right)+g_{0}^{2}}{\left(E-\omega_{0}\right)\left[1-v_{0} G(E)\right]-g_{0}^{2} G(E)}, \quad G(E) \equiv \int \frac{d^{3} \boldsymbol{p}}{(2 \pi)^{3}} \frac{1}{E-p^{2} /(2 \mu)+i 0^{+}},
$$

where $G(E)$ denotes the loop function of the scattering channel and $\mu=M m /(M+m)$. The scattering amplitude $\mathscr{F}(E)$ is given as $\mathscr{F}(E)=-\mu t(E) /(2 \pi)$.

Let us suppose the system to have a discrete eigenstate. The pole position of the amplitude $E=E_{\text {pole }}$ is given as

$$
\left(E_{\text {pole }}-\omega_{0}\right)\left[1-v_{0} G\left(E_{\text {pole }}\right)\right]-g_{0}^{2} G\left(E_{\text {pole }}\right)=0 .
$$

The residue of the pole $g^{2}$ can be given as

$$
g^{2}=\frac{v_{0}\left(E_{\text {pole }}-\omega_{0}\right)+g_{0}^{2}}{1-v_{0}\left[G\left(E_{\text {pole }}\right)+\left(E_{\text {pole }}-\omega_{0}\right) G^{\prime}\left(E_{\text {pole }}\right)\right]+g_{0}^{2} G^{\prime}\left(E_{\text {pole }}\right)},
$$

where $G^{\prime}(E)$ denotes the energy derivative of the loop function $G(E)$.

Now we consider the ZCL where the coupling between the scattering channel and discrete channel vanishes; $g_{0} \rightarrow 0$. Equation ([2.3]) tells us that $E_{\text {pole }}$ moves toward $\omega_{0}$ or $E_{0}$ with $1-$ $v_{0} G\left(E_{0}\right)=0$. If the interaction of the scattering channel is the origin of the eigenstate, the pole remains in the amplitude and its position in the exact ZCL is determined by the dynamics of the scattering channel. Thus the pole moves toward $E=E_{0}$ in this case. On the other hand, if the origin is the eigenstate, the pole must move to $\omega_{0}$. Simultaneously, the residue of the pole in Eq. (2.4) vanishes and the pole decouples from the amplitude.

Next we consider the relation between the CDD zero and the ZCL. The position of the CDD zero can be determined by the zero of the numerator of Eq. (2.2) as

$$
E_{\mathrm{CDD}}=\omega_{0}-g_{0}^{2} / v_{0}
$$

In the ZCL, the CDD zero moves toward $\omega_{0}$. Thus, if the eigenstate originates in the discrete state, the CDD zero and the pole have the same destination. In the exact ZCL, the pole and the CDD zero decouple from the amplitude because the zeros of the numerator and denominator in Eq. (2.2) cancel out each other. The distance between $E_{\mathrm{pole}}$ and $E_{\mathrm{CDD}}$ in this case proportionals to $g_{0}^{2}$. Thus when the coupling $g_{0}$ is small but finite, the CDD zero lies near the pole.

\section{Origin of eigenstate and nearby CDD zero}

In the previous section, we have seen that the CDD zero cancels out the eigenstate pole when the pole decouples from the amplitude. In this section, with the discussion on the topological nature 
of the scattering amplitude, we show that this annihilation of the pole occurs inevitably rather than a coincidence.

Let us consider the topological property of an analytically continued partial-wave scattering amplitude $\mathscr{F}_{i i}(z)$ of channel $i$, where $z$ denotes the complex energy variable. Because $\mathscr{F}_{i i}(z)$ is a meromorphic function of $z$, for a counterclockwise closed contour $C$ on which $\mathscr{F}_{i i}(z)$ is analytic, the argument principle leads to

$$
\frac{1}{2 \pi} \oint_{C} d z \frac{d \arg \mathscr{F}_{i i}(z)}{d z}=n_{Z}-n_{P} \equiv n_{C},
$$

where $n_{Z}$ and $n_{P}$ are integers representing the number of zeros and poles enclosed by the contour $C{ }^{2}$ To derive Eq. (B.D), one can rewrite the integrand as $\left(d \mathscr{F}_{i i}(z) / d z\right) / i \mathscr{F}_{i i}(z) .{ }^{3}$ Because $n_{C}$ is the topological invariant of $\pi_{1}(\mathrm{U}(1)) \cong \mathbb{Z}$, it must be an integer. As an example, if one pole (zero) is enclosed by $C, n_{C}$ equals to $-1(+1)$.

When we gradually reduce the channel coupling toward the ZCL, the poles and zeros move continuously and make the trajectories in the complex $z$ plane. As long as the contour $C$ is chosen not to intersect with the trajectories, the value of $n_{c}$ must be kept because of the topological nature. This means that an abrupt transition from the amplitude with one pole in $C\left(n_{C}=-1\right)$ to nothing $\left(n_{C}=0\right)$ is forbidden. Thus, for a pole to decouple from the amplitude in the ZCL, which corresponds to the case where the eigenstate pole originates in the hidden channel in the previous discussion, it is necessary for the pole to encounter with a nearby zero because the pair annihilation of the pole and the zero is the only way to disappear without changing the value of $n_{C}$.

With the above discussions, we understand that the pole must have the nearby CDD zero if the pole is not dynamically generated in the channel of interest. This means that the origin of the eigenstate can be elucidated as the following manner:

1. If there is no CDD zero near a pole in $\mathscr{F}_{i i}(z)$, the eigenstate originates in channel $i$.

2. If a pole has a nearby CDD zero, the origin of the eigenstate is not in channel $i$.

Thus, just with the position of the eigenstate pole and CDD zero obtained by the analyses of the scattering amplitude, we can specify the origin of the eigenstate.

\section{Application to $\Lambda(1405)$}

We apply the developed method to the $\Lambda(1405)$ baryon resonance and discuss its origin. The two pole structure of $\Lambda(1405)$ [14] is confirmed in the recent analyses of experimental data with next-to-leading order chiral SU(3) dynamics [15, [6]. In the latest version of PDG [四], the pole near the $\bar{K} N(\pi \Sigma)$ threshold is called High-mass (Low-mass) pole.

Utilizing the effective Tomozawa-Weinberg (ETW) model [ए6] with isospin symmetric hadron masses, we study the singularity structure of the $\bar{K} N$ and the $\pi \Sigma$ amplitude. The pole positions are obtained as

$$
W_{\text {pole }}^{\text {Low }}=1375-65 i \mathrm{MeV}, \quad W_{\text {pole }}^{\text {High }}=1423-22 i \mathrm{MeV} .
$$

\footnotetext{
${ }^{2}$ Here we take $C$ so as not to intersect the branch cut.

${ }^{3}$ Here it is assumed that all the poles and zeros are simple. Singularities with multiplicity can appear only with a fine tuning of the parameters, and its existence is not stable against the small perturbation of the parameters.
} 

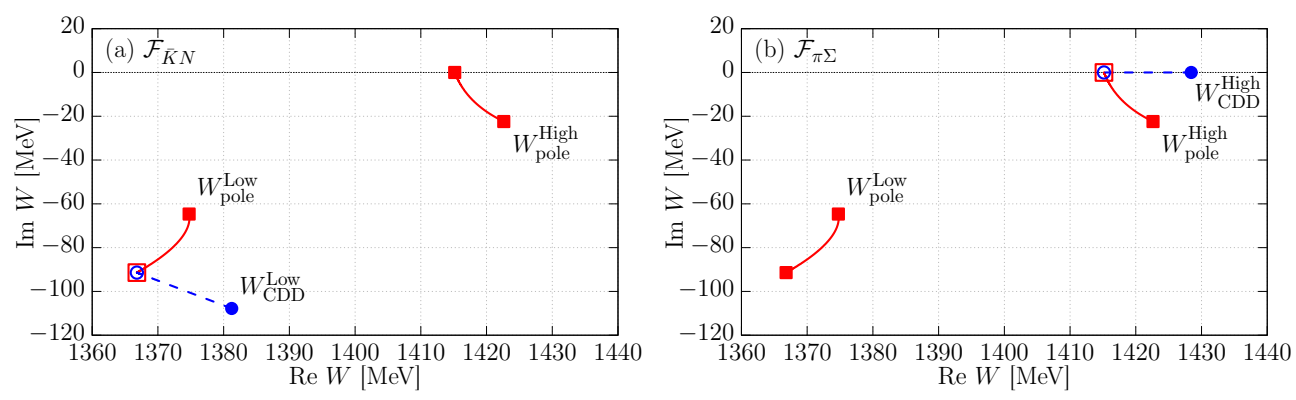

Figure 1: The positions of $W_{\text {pole }}^{\text {Low }}, W_{\text {pole }}^{\text {High }}, W_{\mathrm{CDD}}^{\mathrm{Low}}$ and $W_{\mathrm{CDD}}^{\text {High }}$ in the $\bar{K} N$ amplitude (a) and the $\pi \Sigma$ amplitude (b) quoted from Ref. [U]]. The solid (dashed) line denotes the trajectories of poles (CDD zeros) toward the ZCL.

Searching for the CDD zero in the $\bar{K} N$ and $\pi \Sigma$ amplitude in the $\Lambda(1405)$ energy region, we find one CDD zero in each component. Because the zero in the $\bar{K} N$ amplitude lies close to the Low-mass pole while that in the $\pi \Sigma$ amplitude lies close to the High-mass pole, we denote their energy as $W_{\mathrm{CDD}}^{\mathrm{Low}}$ and $W_{\mathrm{CDD}}^{\mathrm{High}}$, respectively, which are obtained as

$$
W_{\mathrm{CDD}}^{\mathrm{Low}}=1381-108 i \mathrm{MeV}, \quad W_{\mathrm{CDD}}^{\mathrm{High}}=1428-0 i \mathrm{MeV} .
$$

The positions of the poles and CDD zeros are shown in Fig. W.

Now we discuss the origin of the eigenstates with the positions of the pole and CDD zeros. First let us focus on the High-mass pole. In the $\pi \Sigma$ channel, this pole has the accompanied CDD zero, which indicates that the origin of this pole is not the dynamics of the $\pi \Sigma$ channel. On the other hand, absence of the nearby CDD zero in the $\bar{K} N$ channel tells us that the $\bar{K} N$ channel is its origin. In the same manner, we can conclude that the origin of the Low-mass pole is the dynamics of the $\pi \Sigma$ channel because the nearby CDD zero exits only in the $\bar{K} N$ channel.

Finally, to verify the origin of the eigenstate expected in the above discussion, we calculate the trajectories of the poles and the CDD zeros toward the ZCL, as shown in Fig. W. While the Highmass (Low-mass) pole encounters with the High-mass (Low-mass) zero and decouples from the amplitude in the $\pi \Sigma(\bar{K} N)$ channel, it remains in the $\bar{K} N(\pi \Sigma)$ amplitude. This behavior supports that the origin of the High-mass (Low-mass) pole is the dynamics of the $\bar{K} N(\pi \Sigma)$ channel, as concluded with the method constructed in this study.

\section{Summary}

We have discussed the relation between the CDD zeros lying near the eigenstate poles and the origin of the eigenstate. We have shown that the origin is specified with the behavior of the pole in the ZCL of the coupled channel scattering amplitude. If the state has the dynamical origin of the hidden channel, the pole must be annihilated with the nearby CDD pole, whose existence is guaranteed with the topological property of the scattering amplitude. Then we have argued that the origin of the eigenstate can be specified whether the eigenstate pole has the nearby CDD zero or not. By applying this method to $\Lambda(1405)$, it is concluded that the origin of the High-mass (Lowmass) pole is the dynamics of the $\bar{K} N(\pi \Sigma)$ channel. Finally, we emphasize that our method has the broad applicability because it is constructed based on the topological nature of the scattering 
amplitude without any assumption on the interaction. Furthermore, this is a model independent method because what we need to utilize is only the positions of the poles and CDD zeros, which are in principle be uniquely determined from the amplitude. Because of these advantages, this method will shed light on the origin of many hadrons resonances.

\section{References}

[1] Particle Data Group, C. Patrignani et al., Review of Particle Physics, Chin. Phys. C40 (2016) 100001.

[2] J. R. Taylor, Scattering Theory: The quantum Theory on Nonrelativistic Collisions, Wiley 1972.

[3] L. Castillejo, R. H. Dalitz, and F. J. Dyson, Low's scattering equation for the charged and neutral scalar theories, Phys. Rev. 101 (1956) 453.

[4] G. F. Chew and S. C. Frautschi, Potential scattering as opposed to scattering associated with independent particles in the s-matrix theory of strong interactions, Phys. Rev. 124 (1961) 264.

[5] J. A. Oller and E. Oset, N/D description of two meson amplitudes and chiral symmetry, Phys. Rev. D60 (1999) 074023.

[6] U.-G. Meissner and J. A. Oller, Chiral unitary meson baryon dynamics in the presence of resonances: Elastic pion nucleon scattering, Nucl. Phys. A673 (2000) 311 [nucl-th/9912026].

[7] C. Hanhart, Yu. S. Kalashnikova, and A. V. Nefediev, Interplay of quark and meson degrees of freedom in a near-threshold resonance: multi-channel case, Eur. Phys. J. A47 (2011) 101 [1106.1185].

[8] Z.-H. Guo and J. A. Oller, Resonance on top of thresholds: the $\Lambda_{c}(2595)^{+}$as an extremely fine-tuned state, Phys. Rev. D93 (2016) 054014 [1601.00862].

[9] Y. Kamiya and T. Hyodo, Generalized weak-binding relations of compositeness in effective field theory, Prog. Theor. Exp. Phys. 2017 (2017) 023D02 [1607.01899].

[10] Y. Kamiya and T. Hyodo, Structure of hadron resonances with nearby zero of amplitude, (2017) 1711.04558 .

[11] R. J. Eden and J. R. Taylor, Poles and Shadow Poles in the Many-Channel S Matrix, Phys. Rev. 133 (1964) B1575.

[12] B. C. Pearce and B. F. Gibson, Observable Effects of Poles and Shadow Poles in Coupled Channel Systems, Phys. Rev. C40 (1989) 902.

[13] A. Cieply, M. Mai, U.-G. Meissner, and J. Smejkal, On the pole content of coupled channels chiral approaches used for the KNN system, Nucl. Phys. A954 (2016) 17 [1603.02531].

[14] D. Jido, J. A. Oller, E. Oset, A. Ramos, and U. G. Meissner, Chiral dynamics of the two Lambda(1405) states, Nucl. Phys. A725 (2003) 181 [nucl-th/0303062].

[15] Y. Ikeda, T. Hyodo, and W. Weise, Improved constraints on chiral SU(3) dynamics from kaonic hydrogen, Phys. Lett. B706 (2011) 63 [1109.3005].

[16] Y. Ikeda, T. Hyodo, and W. Weise, Chiral SU(3) theory of antikaon-nucleon interactions with improved threshold constraints, Nucl. Phys. A881 (2012) 98 [1201.6549]. 